\title{
Drug Metabolism within the Brain Changes Drug Response: Selective Manipulation of Brain CYP2B Alters Propofol Effects
}

\author{
Jibran Y Khokhar' and Rachel F Tyndale*,' \\ 'Centre for Addiction and Mental Health (CAMH) and Departments of Pharmacology and Toxicology and Psychiatry, University of Toronto, \\ Toronto, Ontario, Canada
}

\begin{abstract}
Drug-metabolizing cytochrome P450 (CYPs) enzymes are expressed in the liver, as well as in extrahepatic tissues such as the brain. Here we show for the first time that drug metabolism by a CYP within the brain, illustrated using CYP2B and the anesthetic propofol (2, 6-diisopropylphenol, Diprivan), can meaningfully alter the pharmacological response to a CNS acting drug. CYP2B is expressed in the brains of animals and humans, and this CYP isoform is able to metabolize centrally acting substrates such as propofol, ecstasy, and serotonin. Rats were given intracerebroventricularly (i.c.v.) injections of vehicle, C8-xanthate, or 8-methoxypsoralen (CYP2B mechanismbased inhibitors) and then tested for sleep time following propofol $(80 \mathrm{mg} / \mathrm{kg}$ intraperitoneally). Both inhibitors significantly increased sleep-time (1.8- to 2-fold) and brain propofol levels, while having no effect on plasma propofol levels. Seven days of nicotine treatment can induce the expression of brain, but not hepatic, CYP2B, and this induction reduced propofol sleep times by 2.5 -fold. This reduction was reversed in a dose-dependent manner by i.c.v. injections of inhibitor. Sleep times correlated with brain $(r=0.76, P=0.0009)$, but not plasma $(r=0.24, P=0.39)$ propofol concentrations. Inhibitor treatments increased brain, but not plasma, propofol levels, and had no effect on hepatic enzyme activity. These data indicate that brain CYP2B can metabolize neuroactive substrates (eg, propofol) and can alter their pharmacological response. This has wider implications for localized CYP-mediated metabolism of drugs, neurotransmitters, and neurotoxins within the brain by this highly variable enzyme family and other CYP subfamilies expressed in the brain. Neuropsychopharmacology (20II) 36, 692-700; doi:I0.1038/npp.2010.202; published online 24 November 2010
\end{abstract}

Keywords: cytochrome P450; brain; propofol; metabolism; sleep; anesthesia

\section{INTRODUCTION}

The liver is the primary site of cytochrome P450 (CYPs) enzyme-mediated drug metabolism (Lewis, 1996); however, several CYP isoforms have also been detected in the brain with variable distribution among different brain regions and expression in both neuronal and glial cell types. Cerebral expression of CYPs might be able to alter metabolism of drugs in a clinically relevant manner (Gervasini et al, 2004), but this has not been shown. Although these brain CYPs are functional in vitro (Albores et al, 2001; Miksys and Tyndale, 2009), it is unclear as to whether these enzymes have sufficient cofactors, coenzymes, and activity in the brain to meaningfully impact local drug metabolism and, by extension, central drug response. The clinical relevance of brain CYP-mediated drug metabolism has not been shown, in part, because of difficulty in determining the relative in vivo contribution of CNS

*Correspondence: Dr RF Tyndale, Department of Pharmacology and Toxicology, I King's College Circle, University of Toronto, Medical Sciences Building, Room 4326, Toronto, Ontario M5S IA8, Canada, Tel: + | 416978 6374, Fax: + | 4169786395 ,

E-mail: r.tyndale@utoronto.ca

Received 20 August 2010; revised 13 October 2010; accepted 25 October 2010 metabolism in the presence of hepatic metabolism and the passage of peripheral metabolites into the brain. Recent advances in our ability to assess the expression and activity of extrahepatic CYPs indicate that rat brain CYPs are active in vivo and metabolism by these CYPs can be altered locally in the brain (Miksys and Tyndale, 2009). This enables us, for the first time, to investigate the impact of CNS CYPs on drug response.

CYP2Bs are a CYP subfamily, members of which are expressed in the brains of rats, mice, monkeys, and humans (Miksys and Tyndale, 2002). CYP2Bs metabolize a variety of CNS acting drugs such as propofol (2, 6-diisopropylphenol, Diprivan) and bupropion, and also play a role in the metabolism of neurochemicals and neurotoxins (Ekins et al, 2008). Rat brain, but not liver, CYP2B can be induced by 7-day nicotine treatment, with a return to baseline levels 7 days later (Khokhar et al, 2010). Both basal and induced CYP2B activity can be inhibited selectively in the brain by an injection of a mechanism-based inhibitor (MBI) also known as a suicide inhibitor (Miksys and Tyndale, 2009).

CYP2B is present in human brain and shows large interindividual variation in expression owing, in part, to genetic polymorphisms (Miksys et al, 2003). In addition, similar to the higher CYP2B brain levels found in nicotine-treated rats, 
human smokers have higher brain CYP2B levels than nonsmokers (Miksys et al, 2003). Interindividual differences in brain CYP2B might alter the metabolism of propofol, thereby contributing to the great variability seen in the response to propofol (Iohom et al, 2007; Kanto and Gepts, 1989). Using a rat model of brain CYP2B manipulation, we investigated the functional consequences of inducing and inhibiting brain CYP2B-mediated metabolism of propofol on drug response. Propofol is a commonly used anesthetic and sedative because of its short duration of action, rapid onset, and preferable side effect and recovery profiles (Langley and Heel, 1988). Upon administration in humans, propofol distributes rapidly and is cleared by both glucuronidation and hydroxylation to the inactive 4-OH metabolite (Favetta et al, 2002). CYP2B6 is the major contributor to the interindividual differences in the rate of propofol hydroxylation (Court et al, 2001). In the rat, CYPmediated hydroxylation is the primary route of metabolism for propofol (Le Guellec et al, 1995).

When the propofol dose was modeled to achieve a desired effect site (brain) concentration, it more accurately predicted the depth of anesthesia compared with modeling for plasma concentrations (Liu et al, 2009). In addition, a hysteresis exists between arterial propofol concentration and anesthetic effect, whereas brain propofol has a close relationship with cerebral blood flow and depth of anesthesia in a sheep model of propofol pharmacokinetics (Ludbrook et al, 1996). In the rat, brain propofol concentrations correlate with tail-flick latency after propofol administration (Shyr et al, 1995), which indicates that the local concentration of propofol in the brain contributes to the drug's effect. Propofol acts primarily on $\mathrm{GABA}_{\mathrm{A}}$ receptors (Altomare et al, 2003); it can also inhibit some subtypes of nicotinic acetylcholine receptors (nAChRs), but they are not the primary sites of action for the drug (Tassonyi et al, 2002). Repeated exposure to propofol does not result in tolerance (Fassoulaki et al, 1994), making this model suitable for multiple testing in a within-animal design.

Here, we use a rat model of propofol sedation to study the effects of altered brain, and not hepatic, CYP2B-mediated metabolism on propofol sleep time. More broadly, this study is a proof of concept for the contribution of central metabolism, in addition to hepatic metabolism, to altered response to CNS acting drugs. This mechanism could help clarify a disconnect which is often seen between plasma drug levels and central drug effects, as well as further our understanding of interindividual differences in drug response.

\section{MATERIALS AND METHODS}

\section{Animals}

Adult male Wistar rats (250-300 g; Charles River, St-Constant, $\mathrm{PQ}$, Canada) were housed in pairs and kept under a 12-h artificial light/dark cycle (lights on at 0600 hours). The animals were handled daily to habituate them to manipulation. All procedures were approved by the Animal Care Committee at the University of Toronto.

\section{Drug Treatment}

Propofol (10 mg/ml Diprivan; AstraZeneca, Mississauga, ON, Canada) was injected intraperitoneally (i.p.) at $40-120 \mathrm{mg} / \mathrm{kg}$ for the dose-response experiments, $80 \mathrm{mg} / \mathrm{kg}$ for the inhibition and induction experiments, and 40 and $50 \mathrm{mg} / \mathrm{kg}$ for the dose-response shift experiments. C8-xanthate (C8-X) was purchased from Toronto Research Chemicals (Toronto, ON, Canada) and was dissolved in artificial cerebro-spinal fluid (ACSF), and $0.625-80 \mu \mathrm{g}$ was given intracerebroventricularly (i.c.v.) in a $0.5-2 \mu \mathrm{l}$ total volume. A dose of $40 \mu \mathrm{g}$ radiolabeled MBI 8-methoxypsoralen $\left({ }^{3} \mathrm{H}-8-\mathrm{MOP}\right)$ (a gift from William $\mathrm{F}$ Trager) was dissolved in $0.5 \mu \mathrm{l} \mathrm{ACSF}$ and given i.c.v.. Nicotine bitartarate was purchased from Sigma-Aldrich Canada (Oakville, ON, Canada) and rats were injected subcutaneously (s.c.) once daily for 7 days with $1 \mathrm{mg}$ nicotine base per kg body weight in sterile saline ( $\mathrm{pH} 7.4)$.

\section{Inhibition of Rat Brain CYP2B Activity Following I.C.V. Injection of a CYP2B MBI}

The methods used were modified from previous studies (Khokhar et al, 2010; Miksys and Tyndale, 2009). Briefly, the rats were treated for 7 days with nicotine $(1 \mathrm{mg} / \mathrm{kg})$ or saline. The rats were anesthetized (isoflurane) and placed in the stereotaxic frame. The animals then received an i.c.v. injection into the right lateral ventricle (Bregma coordinates: dorsal-ventral, -3.6 ; anterior-posterior, -0.9 ; lateral, -1.4; Paxinos and Watson, 1986) of either $20 \mu \mathrm{g}$ C8-X (CYP2B MBI; Yanev et al, 2000) in $0.5 \mu \mathrm{l}$ ACSF or ACSF alone (vehicle). All i.c.v. injections were made over $2 \mathrm{~min}$, and the Hamilton syringe was left in place for 3 min postinjection. At $20 \mathrm{~h}$ after the first i.c.v. injection, the rats received another i.c.v. injection of $40 \mu \mathrm{g}$ of ${ }^{3} \mathrm{H}-8$-MOP in $0.5 \mu \mathrm{l}$ sterile ACSF. The animals were killed $4 \mathrm{~h}$ after the ${ }^{3} \mathrm{H}-8$-MOP injection to assess the remaining rat brain CYP2B activity $24 \mathrm{~h}$ after i.c.v. injection of the inhibitor $\mathrm{C} 8-\mathrm{X}$.

As described previously (Miksys and Tyndale, 2009), $400 \mu \mathrm{g}$ brain membranes from animals killed as described above were incubated overnight with $100 \mu \mathrm{l}$ of monoclonal antibody raised against rat CYP2B1/2 (Fitzgerald Industries, Concord, MA) in phosphate-buffered saline (PBS, pH 7.4) at $4^{\circ} \mathrm{C}$. The antibody-CYP2B protein complex was then incubated with $400 \mu \mathrm{l}$ of a $50 \%$ slurry of protein G immobilized on resin beads (Pierce, Rockford, IL) for $3 \mathrm{~h}$ on a rocker and then centrifuged for $1 \mathrm{~min}$ at $3000 \mathrm{~g}$. The beads were washed twice with $500 \mu \mathrm{l}$ PBS, centrifuged for $1 \mathrm{~min}$ at $3000 \mathrm{~g}$, and then re-suspended in PBS. Aliquots of the beads and supernatants were counted. In vivo brain CYP2B activity is expressed as the percentage of radioactive counts bound to the beads relative to total counts detected in $400 \mu \mathrm{g}$ of brain membranes. To further assess the specificity of the MBI for rat brain CYP2B, $100 \mu \mathrm{l}$ of monoclonal antibodies raised against CYP2A, CYP2D, CYP2E, and CYP3A (BD Biosciences, Mississauga, ON, Canada) were also incubated with $400 \mu \mathrm{g}$ of radiolabeled frontal cortex (FC) membranes as outlined above for the antibody raised against CYP2B.

\section{Rat Hepatic CYP2B Activity Measured by In Vitro Nicotine Oxidation}

Rat hepatic CYP2B activity was measured using in vitro nicotine oxidation, as this is a sensitive and selective assay for rat CYP2B (Nakayama et al, 1993). Rat liver microsomal 
proteins were prepared as done previously (Siu and Tyndale, 2008) from rats that received an i.c.v. injection of MBI $(20 \mu \mathrm{g}$ C8-X or $40 \mu \mathrm{g} 8$-MOP) or vehicle control (ACSF). As before (Siu and Tyndale, 2008), microsomes $(0.5 \mathrm{mg} / \mathrm{ml})$ were incubated with nicotine (a CYP2B substrate; 120 and $480 \mu \mathrm{M}), 1 \mathrm{mM} \mathrm{NADPH}$, and $1.5 \mathrm{mg} / \mathrm{ml}$ liver cytosol in $50 \mathrm{mM}$ Tris- $\mathrm{HCl}$ buffer ( $\mathrm{pH}$ 7.4) for $20 \mathrm{~min}$ at $37^{\circ} \mathrm{C}$ in a final volume of $0.5 \mathrm{ml}$. The reaction was stopped using $4 \%(\mathrm{v} / \mathrm{v}) \mathrm{Na}_{2} \mathrm{CO}_{3}$. After incubation, the internal standard 5-methylcotinine $(65 \mu \mathrm{g})$ was added with $50 \mu \mathrm{l}$ of $\mathrm{NaOH}(10 \mathrm{M})$ and $4 \mathrm{ml}$ of dichloromethane. The tubes were capped and shaken for $10 \mathrm{~min}$, and then centrifuged at 3000 r.p.m. for $10 \mathrm{~min}$. The organic fraction was added to $25 \mu \mathrm{l}$ of $6 \mathrm{~N} \mathrm{HCl}$, evaporated under a nitrogen stream at $37^{\circ} \mathrm{C}$, and dissolved in $105 \mu \mathrm{l}$ of distilled water. A $90 \mu \mathrm{l}$ aliquot was analyzed by HPLC-UV, with a limit of quantification of $5 \mathrm{ng} / \mathrm{ml}$ for both nicotine and cotinine.

\section{Dose-Response Curve for Sleep Time Following an Intraperitoneal Injection of Propofol}

Rats were given $40-120 \mathrm{mg} / \mathrm{kg}$ propofol i.p. and were then placed under a heat lamp and monitored for propofolinduced sedation. Sleep was defined as the loss of purposeful movement, with the end point being the initial moment of head lift (Dam et al, 1990). Blood draws to measure plasma propofol levels or killings were performed $2 \mathrm{~h}$ after propofol injection. The livers and brains were removed and frozen from killed animals for measurement of hepatic CYP2B activity and brain propofol levels; no perfusion was performed before killings to avoid differences in collection of blood for propofol analyses throughout the study. All experiments, unless otherwise indicated, were performed in a within-animal design.

\section{Effect of I.C.V. MBI Injection on Propofol-Induced Sedation}

Propofol-induced sleep times were measured at baseline and $24 \mathrm{~h}$ after an i.c.v. injection of a CYP2B MBI $(20 \mu \mathrm{g}$ C8-X or $40 \mu \mathrm{g} 8$-MOP) or the vehicle control (ACSF). C8-X and 8-MOP had similar effects, but C8-X has greater selectivity for CYP2B (Yanev et al, 1999), hence, C8-X was used for all further experiments.

\section{Effects of Nicotine's Induction of Rat Brain CYP2B and nAChR Blockade on Propofol Response}

Rats were tested for baseline propofol response and were then injected s.c. once daily for 7 days with nicotine bitartarate $(1 \mathrm{mg} / \mathrm{kg})$ or the vehicle (saline). The animals were tested for propofol response $24 \mathrm{~h}$ after 1 and 7 days of nicotine treatment. At this dose, nicotine is cleared from the plasma by $24 \mathrm{~h}$ and any acute effects of nicotine on nAChRs may have partially recovered, diminishing any potential confounding effects that the nicotine treatment could have on propofol's actions. CYP2B is still induced $24 \mathrm{~h}$ after 7 -day nicotine treatment in the rat brain (Figure 1b).

After propofol testing post 7-day nicotine treatment, animals were either killed $2 \mathrm{~h}$ after propofol or received another nicotine injection. At $24 \mathrm{~h}$ after the eighth nicotine injection, animals received an i.c.v. injection of $20 \mu \mathrm{g}$ C8-X.
The animals then received a ninth injection of nicotine and were tested with propofol $24 \mathrm{~h}$ later.

As nAChRs are the primary site of action for nicotine in the brain and can be upregulated by chronic nicotine (Vallejo et al, 2005), we assessed whether nAChR blockade altered propofol response, both alone and following 7-day nicotine treatment to determine whether nAChR upregulation affects propofol response. We tested the effects of irreversible (chlorisondamine $10 \mathrm{mg} / \mathrm{kg}$ s.c.) or acute (mecamylamine $1 \mathrm{mg} / \mathrm{kg}$ s.c.) blockade of nAChRs on response to propofol. Chlorisondamine is a quasi-irreversible blocker of nAChRs and blocks nicotine-induced
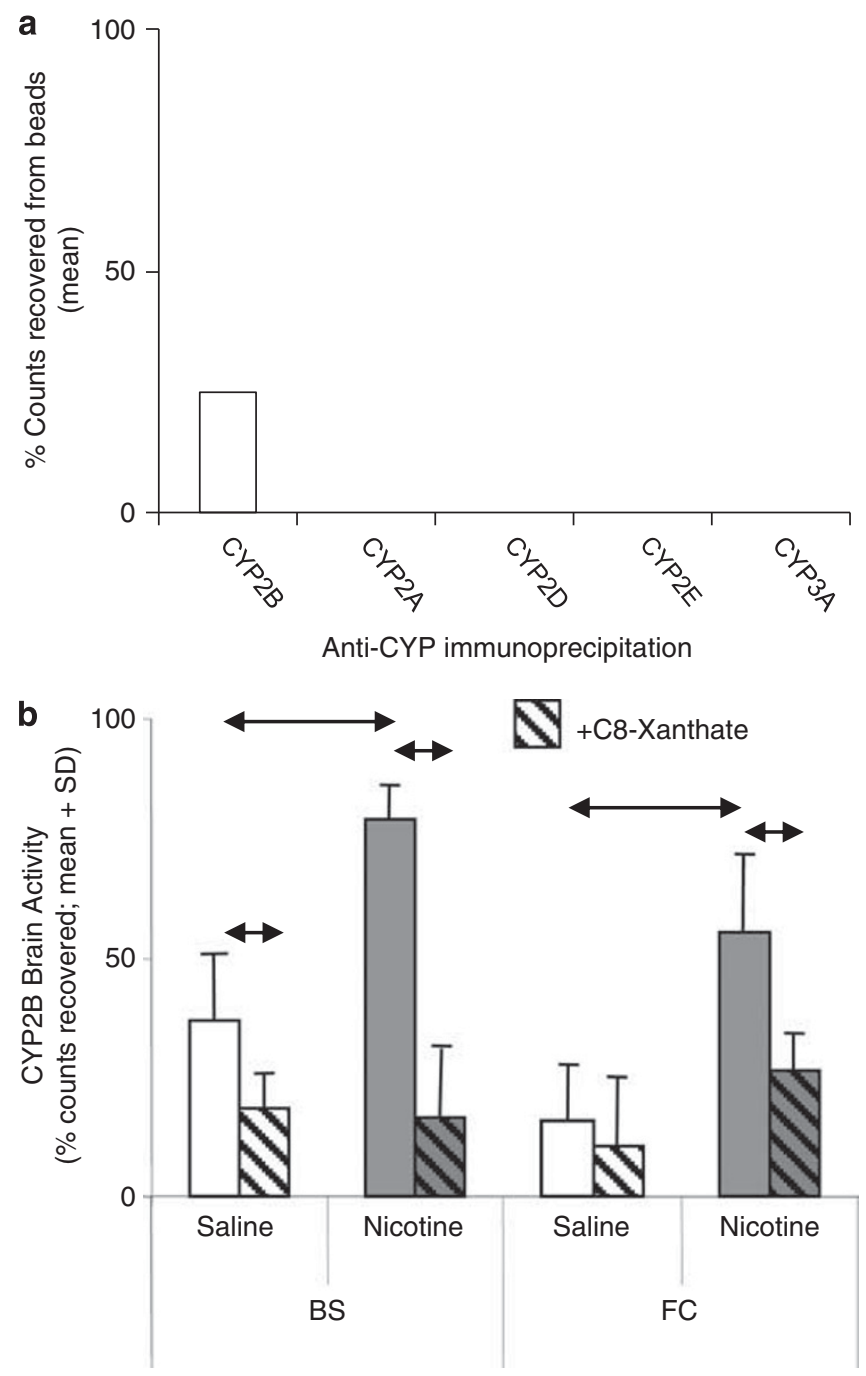

Figure I Selective inhibition of rat brain CYP2B activity after intracerebroventricular (i.c.v.) injection of CYP2B mechanism-based inhibitor (MBI). (a) Radioactivity was detected following immunoprecipitation of rat brain frontal cortex tissue using antibodies specific to CYP2B, but not to CYP2A, CYP2D, CYP2E, or CYP3A, indicating a specificity of MBI activation and irreversible binding to CYP2B enzyme following i.c.v. injection of radiolabeled MBI. (b) Rat brain CYP2B activity was significantly increased by 7-day nicotine treatment in the brainstem $(\leftrightarrow P=0.006)$. Both basal $(\leftrightarrow P=0.05)$ and nicotine-induced CYP2B $(\leftrightarrow P=0.03)$ activity in the brain were significantly reduced in animals pretreated with i.c.v. C8-xanthate (C8-X) compared with the i.c.v. artificial cerebro-spinal fluid (ACSF) pretreated animals in the brainstem. In the frontal cortex, which has low basal activity, 7-day nicotine treatment also increased CYP2B activity ( $\leftrightarrow P=0.04)$ and pretreatment with i.c.v. C8-X inhibited this ( $\leftrightarrow P=0.05$ ). 
responses for up to 2 weeks (Clarke et al, 1994); chlorisondamine pretreatment does not alter CYP2B induction by nicotine (Khokhar et al, 2010). Propofol response was measured at baseline and after chlorisondamine pretreatment. The animals then received 7-day nicotine treatment and were tested with propofol $24 \mathrm{~h}$ after the last nicotine injection. The mecamylamine study was run similarly, but the animals received mecamylamine $30 \mathrm{~min}$, as described previously (Bhargava and Saha, 2001), before every propofol test (except baseline measurements).

\section{Dose-Dependent Reversal of Nicotine-Induced Sleep- Time Reductions by CYP2B C8-X I.C.V. Injections}

Animals were tested with propofol: at baseline, after a 7-day nicotine treatment, and $24 \mathrm{~h}$ after receiving an eighth injection of nicotine and an i.c.v. injection $(0-80 \mu \mathrm{g} C 8-\mathrm{X}$, $n=4$ per C8-X dose group, 12/ACSF group).

\section{Rat Brain CYP2B Alteration Can Shift Propofol's Dose-Response Curve}

Rats were tested with low doses of propofol ( 40 and $50 \mathrm{mg} / \mathrm{kg}$ ) at baseline and after i.c.v. injection of C8-X $(20 \mu \mathrm{g})$. The animals were tested again with propofol $(50 \mathrm{mg} / \mathrm{kg})$ after 7-day nicotine treatment $(1 \mathrm{mg} / \mathrm{kg}$ s.c.) and after i.c.v. C8-X $(20 \mu \mathrm{g})$.

\section{Measurement of Plasma and Brain Propofol Levels}

Plasma and brain levels of propofol were quantified by HPLC, essentially as before (Seno et al, 2002). Acetonitrile $(100 \mu \mathrm{l})$ and $5 \mu \mathrm{l}$ of thymol $(100 \mu \mathrm{g} / \mathrm{ml}$, internal standard) were added to $100 \mu \mathrm{l}$ of plasma or brain homogenate (brain tissue homogenized in 1:2 (w/v) in $0.2 \mathrm{M}$ phosphate buffer), and mixed by a vortex and centrifuged at 13500 r.p.m. for $20 \mathrm{~min}\left(4^{\circ} \mathrm{C}\right)$. The supernatant $(100 \mu \mathrm{l})$ was injected into the HPLC. The limit of quantification was $5 \mathrm{ng} / 100 \mu \mathrm{l}$ and the assay was linear up to $5 \mu \mathrm{g} / \mathrm{ml}$ with an extraction efficiency of 95\%. The HPLC-UV system (Agilent 1200 Separation Module) was set for detection at $270 \mathrm{~nm}$ and the propofol and internal standard were separated on an Agilent ZORBAX Bonus-RP Column $\left(150 \times 4.6 \mathrm{~mm}^{2}\right.$ I.D.; particle size, $5 \mu \mathrm{m}$ ). The retention times were $6.2 \mathrm{~min}$ for thymol and $12.4 \mathrm{~min}$ for propofol. The mobile phase used was $40 \%$ methanol: acetonitrile $(70: 30(\mathrm{v} / \mathrm{v}))$, and the flow rate was $1.2 \mathrm{ml} / \mathrm{min}$.

\section{Statistical Analyses}

For the within-region, between-treatment group comparisons of brain CYP2B in vivo activity, and the comparison of brain and plasma propofol levels between groups, a oneway ANOVA and Bonferroni correction were used. Pre(baseline) and post-treatment mean sleep times were derived from animal sleep times; means were compared in a paired two-tailed $t$-test (owing to the within-animal design). The nAChR blockade experiment, dose-dependent inhibition experiment, and the shift in propofol doseresponse curve experiment were all tested using repeated measures ANOVA and Bonferroni correction.

\section{RESULTS}

Selective Inhibition of Rat Brain CYP2B Activity after an Intracerebroventricular Injection of a CYP2B MBI

Using a technique that we have developed for establishing in vivo CYP activity in rat brain (Miksys and Tyndale, 2009), we assessed in situ CYP2B activity by measuring the binding of ${ }^{3} \mathrm{H}-8-\mathrm{MOP}$ (Koenigs and Trager, 1998) given i.c.v. to living animals. Radioactivity was detected following immunoprecipitation with antibodies specific to CYP2B, but not to CYP2A, CYP2D, CYP2E, or CYP3A, indicating specificity of radiolabeling for the CYP2B enzyme following i.c.v. injection of the radiolabeled MBI in this assay. CYP2B activity (radioactive counts from enzyme-inhibitor complex immunoprecipitated using CYP2B-specific antibody) was detectable in both brainstem (BS) and FC and was significantly increased by a 7-day nicotine treatment $(P=0.005$ and 0.04 , respectively; Figure $1 \mathrm{~b})$, but no radioactive counts were detected in rat liver microsomes (data not shown). Basal and nicotine-induced CYP2B activity was reduced in animals pretreated with i.c.v. C8-X (another CYP2B MBI; Yanev et al, 1999) compared with the animals pretreated with i.c.v. ACSF. This indicates that the i.c.v.-delivered MBIs bound irreversibly to active CYP2B enzyme in disparate regions of the brain.

\section{Sleep-Time Dose-Response Curve for Propofol}

A dose response for sleep time was observed from 40 to $120 \mathrm{mg} / \mathrm{kg}$ propofol given i.p.. No sleep time was observed at the $40 \mathrm{mg} / \mathrm{kg}$ dose, whereas $120 \mathrm{mg} / \mathrm{kg}$ was fatal to $31 \%$ of the animals (four of 13 tested). At $80 \mathrm{mg} / \mathrm{kg}$ propofol, all of the animals slept, and they had an average sleep time of $30 \pm 8$ min (mean $\pm S D$ ), allowing for measurement of both increases and decreases in sleep time.

\section{I.C.V. Injection of CYP2B MBIs Extends Propofol-Induced Sleep Time}

Repeating propofol tests $(80 \mathrm{mg} / \mathrm{kg}$ i.p.) did not alter sleep time (during baseline trials), indicating no formation of tolerance as observed previously (Fassoulaki et al, 1994). I.C.V. injections of CYP2B MBIs (C8-X and 8-MOP) significantly $(P=0.02$ and $0.04 ; n=6 /$ group $)$ increased propofol sleep time, whereas the ASCF vehicle control did not (Figure 2a and $b$ ). The sleep times correlated with brain propofol levels ( $r=0.76, P=0.0009$; Figure $2 \mathrm{c})$, but not with plasma propofol levels $(r=0.24, P=0.39$; Figure $2 \mathrm{~d})$. This is consistent with the CNS metabolism, and resulting propofol levels, being responsible for the duration of sleep response. Brain propofol levels were significantly higher in the C8-X$(P=0.01)$ and 8 -MOP-treated animals $(P=0.04)$ compared with ACSF-treated controls (Figure 2e). The CNS MBI treatments did not alter plasma propofol concentrations (Figure 2f). There was no difference in the rates of hepatic CYP2B-mediated metabolism ex vivo between animals that received the vehicle (ACSF) or the i.c.v. MBIs C8-X and 8-MOP (Figure 2g). Taken together, the lack of impact of the CNS inhibitor injections on plasma propofol levels and on ex vivo hepatic CYP2B activity indicates that the MBIs given i.c.v. did not reach the liver at concentrations which altered hepatic CYP2B activity or propofol metabolism. 

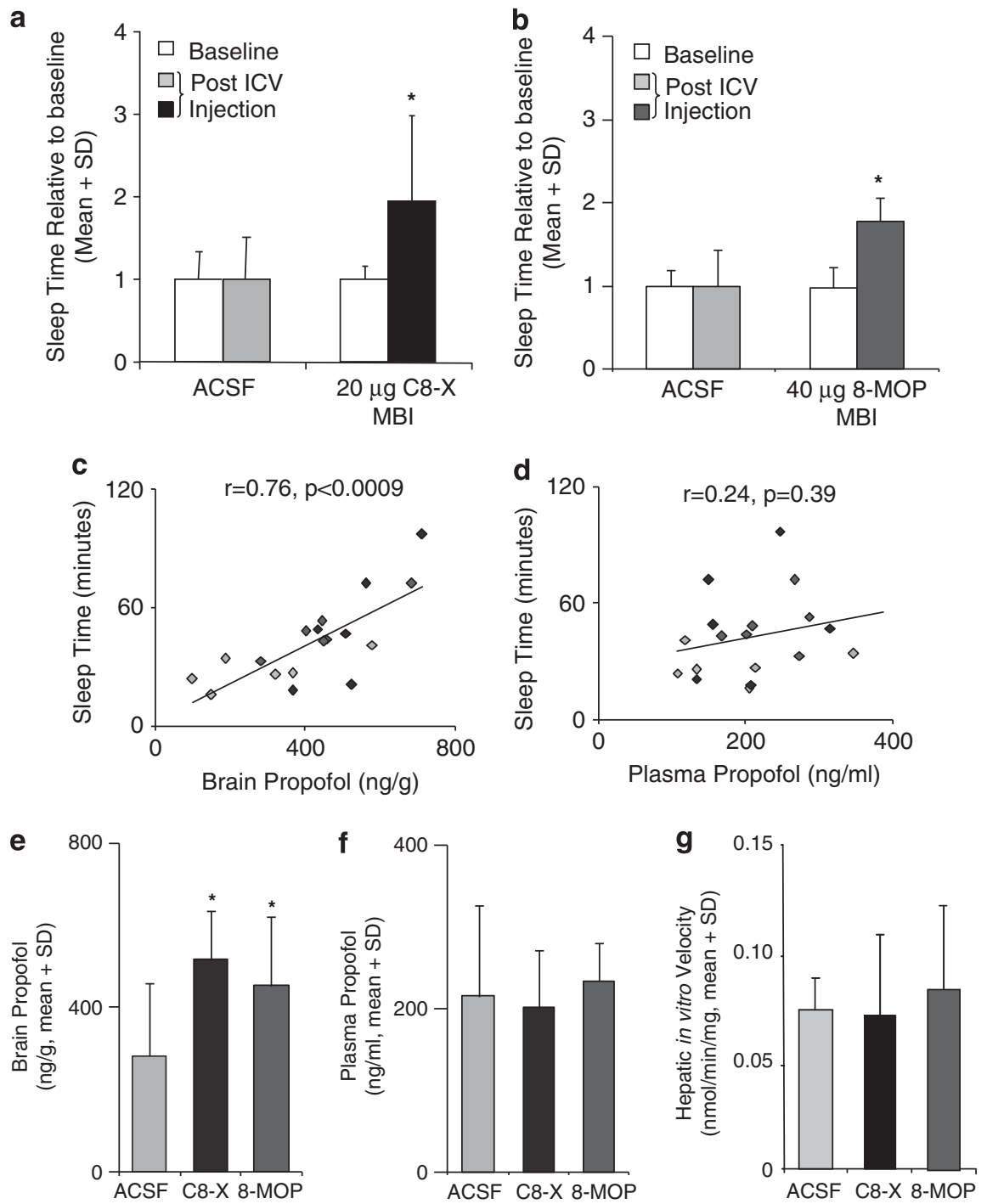

Figure 2 Intracerebroventricular (i.c.v.) injection of CYP2B mechanism-based inhibitor (MBI) extends propofol-induced sleep time and increases brain propofol concentration. I.c.v. injection of the CYP2B MBIs, (a) $20 \mu \mathrm{g}$ C8-xanthate (C8-X) (*P=0.02) and (b) $40 \mu \mathrm{g}$ radiolabeled MBI 8-methoxypsoralen $(8-M O P)(* P=0.04)$, significantly increased propofol sleep time, whereas i.c.v. injections of artificial cerebro-spinal fluid (ACSF) (vehicle control) did not alter sleep time. (c) Sleep times correlated with propofol levels in the brain (includes ACSF, C8-X, and 8-MOP animals), but not with (d) plasma propofol levels (gray: ACSF; black: C8-X; dark gray: 8-MOP). (e) Brain propofol levels were significantly higher in the C8-X $(* P=0.0 \mathrm{I})$ - and $8-\mathrm{MOP}(* P=0.04)$-treated animals when compared with the ACSF controls. (f) No differences in plasma propofol concentrations were found following either MBI treatment. (g) There was no effect of either CYP2B MBI on in vitro hepatic CYP2B-mediated nicotine metabolism; there were no differences in ex vivo nicotine oxidation rates between i.c.v. ACSF and MBls C8-X or 8 -MOP $(0.07 \pm 0.02,0.07 \pm 0.04$, and $0.08 \pm 0.04 \mathrm{nmol} / \mathrm{min} / \mathrm{mg}$, respectively), indicating no detectable effect of MB injected i.c.v. on hepatic CYP2B metabolic activity.

\section{Nicotine Treatment Induces CYP2B and Reduces Propofol-Induced Sleep Regardless of nAChR Blockade}

When animals were tested with propofol $24 \mathrm{~h}$ after a single nicotine injection, which does not induce CYP2B (Khokhar et al, 2010), there was no difference in sleep time compared with baseline ( $21 \pm 4$ vs $23 \pm 3 \mathrm{~min} ; P=0.42)$. After a 7-day nicotine treatment, which induces brain CYP2B (Figure 1b), there was a significant $(P<0.0001)$ reduction in sleep time to $\sim 40 \%$ of baseline ( $n=52$; Figure $3 \mathrm{a})$. Rats were then given i.c.v. C8-X and the reduction in sleep time due to nicotine treatment was reversed and sleep time was extended to $\sim 180 \%$ of baseline $(P=0.02 ; n=4$; Figure $3 b)$. Once again we saw a significant correlation between sleep-time and brain $(r=0.72, P=0.02)$, but not plasma $(r=0.24$, $P=0.38$ ), propofol concentrations. To investigate whether alterations in the nAChRs following the 7-day nicotine treatments contributed to the reduced sleep times postnicotine, we pretreated the animals with an irreversible nAChR blocker chlorisondamine or an acute antagonist mecamylamine. Neither pretreatment altered propofol sleep time compared with baseline $(P>0.05)$ nor the degree of reduction seen following nicotine treatment $(P>0.05)$; the i.c.v. C8-X was still able to reverse the reduction in sleep times $(P<0.05 ; n=6 /$ group; Figure $3 \mathrm{c}$ and d). This suggests that nAChR blockade does not alter propofol response and 
a

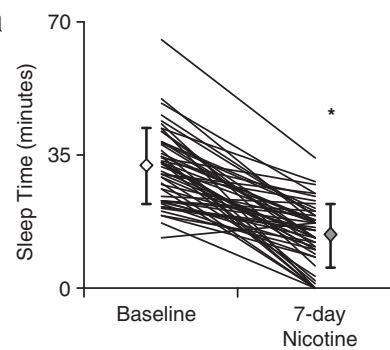

C

Baseline

Post-Chlorisondamin 7-day Nicotine
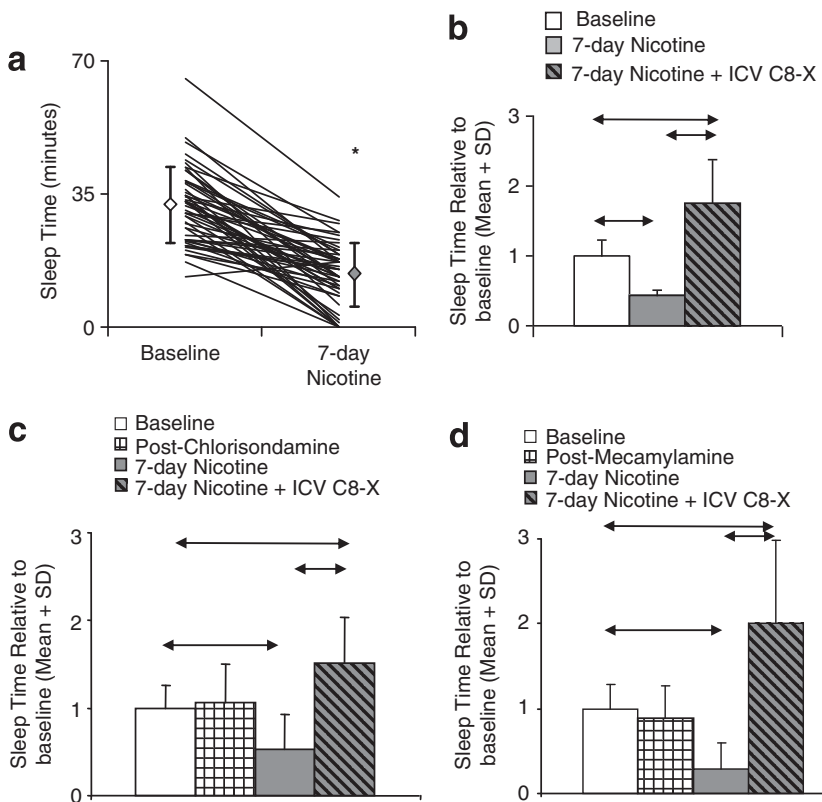

d
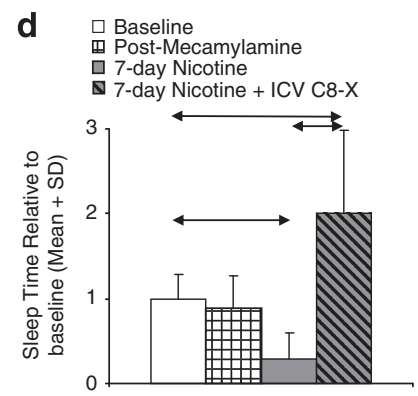

Figure 3 Nicotine-induced CYP2B reduces propofol-induced sleep time, with no effect of nicotinic acetylcholine receptors ( $n A C h R$ ) blockade on this response. (a) Seven-day nicotine treatment significantly reduced mean sleep time by $60 \%$ compared with the animals' sleep time at baseline $(n=52, * P<0.000 \mathrm{I})$. Diamonds represent mean sleep time \pm standard deviation. (b) An intracerebroventricular (i.c.v.) injection of C8-xanthate $(\mathrm{C} 8-\mathrm{X})$ reversed the reduction in sleep time owing to nicotine treatment and sleep time was extended beyond the baseline sleep times $(P=0.02$; $n=4)$. (c) Pretreatments with the $n A C h R$ blocker chlorisondamine and (d) the nAChR antagonist mecamylamine did not alter propofol sleep at baseline or the reduction in sleep time following nicotine treatment, and i.c.v. C8-X reversed the reduction in both groups $(\leftrightarrow P<0.05 ; n=6$ / group).

that the reduction in sleep time after chronic nicotine is not owing to alterations in $\mathrm{nAChR}$ status or interactions between the CYP2B, C8-X, and nAChRs.

\section{I.C.V. Injections of C8-X Dose Dependently Reverse Nicotine-Induced Reductions in Sleep Time}

Low doses of i.c.v. C8-X (1.25-10 $\mu \mathrm{g})$ reversed the effects of nicotine treatments on propofol sleep times, while $0.625 \mu \mathrm{g}$ had no effect (Figure 4a). Doses between 20 and $80 \mu \mathrm{g}$ not only reversed the reduction in sleep times caused by the 7 -day nicotine treatment, but also significantly extended it beyond baseline sleep times $(P<0.05 ; n=4 / \mathrm{C} 8-\mathrm{X}$ group, $n=12 \mathrm{ACSF}$ ); in vitro hepatic CYP2B nicotine metabolism was not altered by these doses of i.c.v. C8-X (0.65 \pm 0.04 , $0.62 \pm 0.08,0.69 \pm 0.06$, and $0.70 \pm 0.09 \mathrm{nmol} / \mathrm{min} / \mathrm{mg}$ for $0,20,40$, and $80 \mu \mathrm{g} C 8-\mathrm{X}$, respectively). There was a dosedependent effect of i.c.v. C8-X on propofol levels in the rat brain with i.c.v. C8-X between 5 and $80 \mu \mathrm{g}$, resulting in significantly higher brain propofol levels than the ACSF control $(P=0.02$; Figure $4 \mathrm{~b})$. In addition, the mean brain propofol levels in the ACSF-treated animals post-nicotine were significantly lower $(P=0.04)$ than animals that did not receive nicotine (Figure 2e), consistent with the reduced sleep times seen after nicotine treatment. Once again, the sleep times correlated with brain $(r=0.67, P=0.01$; Figure $4 \mathrm{~b}$, inset), but not plasma $(r=-0.03, P=0.84)$ a
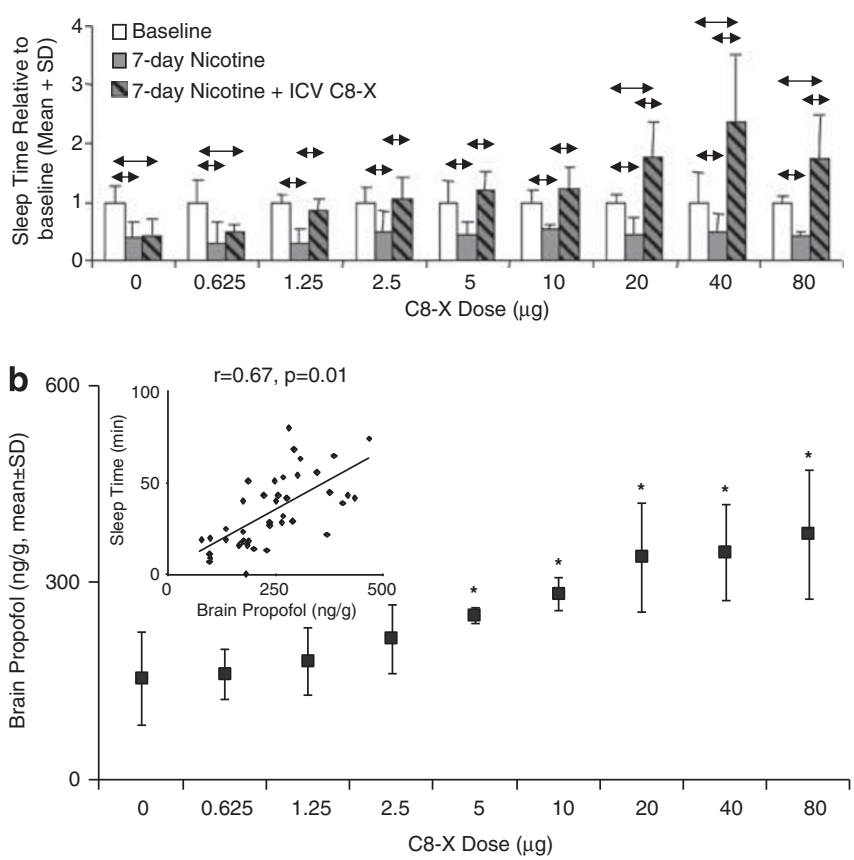

Figure 4 Rat brain CYP2B inhibition dose dependently reverses nicotine-induced sleep-time reduction. (a) A low dose of C8-xanthate $(\mathrm{C} 8-\mathrm{X})(0.625 \mu \mathrm{g})$ did not reverse nicotine-induced reduction in sleep times, whereas $1.25-10 \mu \mathrm{g}$ of $\mathrm{C} 8-\mathrm{X}$ given intracerebroventricularly (i.c.v.) reversed the effects of nicotine treatment returning the sleep times to those seen at baseline. Doses of C8-X between 20 and $80 \mu \mathrm{g}$ not only reversed the reduction in sleep time after 7-day nicotine treatment, but also significantly extended it beyond baseline sleep times $(n=4 /$ group per C8-X dose, $n=12 /$ ACSF group; $\leftrightarrow P<0.05$ ). (b) CYP2B inhibition dose dependently increased rat brain propofol concentration with doses between 5 and $80 \mu \mathrm{g}$ of $\mathrm{C} 8-\mathrm{X}$, resulting in significantly higher brain propofol levels compared with the animals that received $0 \mu \mathrm{g} C 8-\mathrm{X}$ $\left({ }^{*} P<0.05\right)$. Inset: Sleep times were correlated with brain propofol levels and not with plasma propofol levels $(r=-0.03, P=0.84)$.

propofol levels. No differences in daily sleeping or feeding behaviors were seen after either nicotine or i.c.v. inhibitor treatments.

\section{Inhibition or Induction of Rat Brain CYP2B can Shift Propofol's Dose-Response Curve}

A low dose of propofol, $40 \mathrm{mg} / \mathrm{kg}$, was inactive in all animals with a mean sleep time of 0 min (Figure 5), but was made active after an i.c.v. C8-X injection. The animals displayed significantly $(P=0.009)$ longer sleep times, with a mean sleep time of $9 \pm 10 \mathrm{~min}$ (apparent dose: $64 \mathrm{mg} / \mathrm{kg}$ ). A dose of $50 \mathrm{mg} / \mathrm{kg}$ resulted in only five of nine animals sleeping at baseline, with a mean sleep time of $5 \pm 6 \mathrm{~min}$ (Figure 5). Following i.c.v. C8-X, the animals displayed significantly $(P=0.02)$ longer sleep times $(19 \pm 12 \mathrm{~min})$ and seven of nine animals slept (Figure 5). To assess whether this active dose of $50 \mathrm{mg} / \mathrm{kg}$ propofol can be made inactive by increasing brain CYP2B, we treated these animals with nicotine for 7 days to induce brain CYP2B. This treatment abolished the propofol sleep response completely, with no animals exhibiting sleep resulting in an average sleep time of $0 \mathrm{~min}$ $(P=0.04$; Figure 5). An i.c.v. injection of $\mathrm{C} 8-\mathrm{X}$ following nicotine's induction of brain CYP2B reversed the reduction 


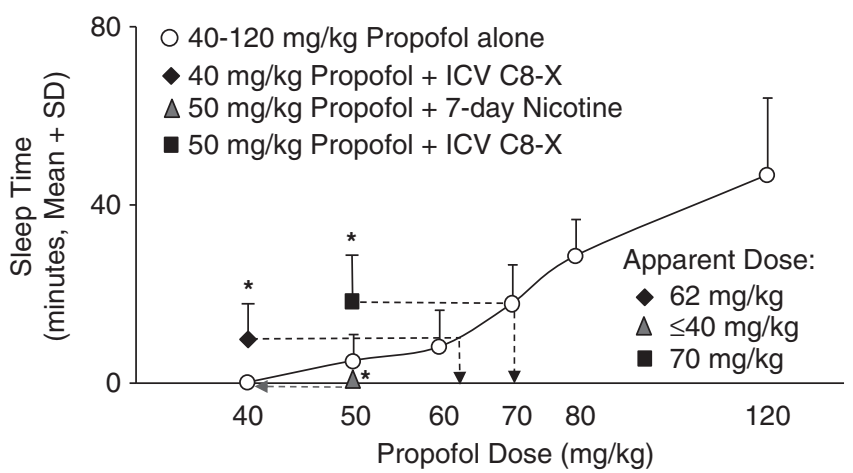

Figure 5 Rat brain CYP2B manipulation shifts the propofol sleep-time dose-response curve. A dose of $40 \mathrm{mg} / \mathrm{kg}$ was inactive at baseline-none of the animals slept (sleep time: $0 \mathrm{~min}$ ). An intracerebroventricular (i.c.v.) injection of C8-xanthate (C8-X) significantly increased sleep time consequently rendering the $40 \mathrm{mg} / \mathrm{kg}$ dose active (sleep time: $9 \mathrm{~min}$; apparent dose: $62 \mathrm{mg} / \mathrm{kg} ; P=0.009 ; n=9$ ). Upon administration of a $50 \mathrm{mg} / \mathrm{kg}$ dose of propofol, the animals slept an average of $\sim 5 \mathrm{~min}$ at baseline. Induction of brain CYP2B activity by 7-day nicotine treatment abolished the propofol response - none of the animals slept (sleep time: $0 \mathrm{~min}$; apparent dose: $40 \mathrm{mg} / \mathrm{kg} ; P=0.02 ; n=9$ ). After i.c.v. C8-X, the rats displayed significantly longer sleep times (sleep time: $19 \mathrm{~min}$; apparent dose: $70 \mathrm{mg} / \mathrm{kg} ; P=0.04 ; n=9$ ). Dashed arrows indicate apparent dose (graph is plotted on a $\log x$ axis, but values on $x$ axis are actual doses).

in sleep time and resulted in significantly greater sleep times (23 $\pm 14 \mathrm{~min}$, eight of nine animals slept) compared with the sleep times at baseline and after nicotine treatment $(P=0.01)$. The sleep times resulting from $50 \mathrm{mg} / \mathrm{kg}$ propofol following nicotine treatment and i.c.v. C8-X were equivalent to propofol doses of 40 and $70-75 \mathrm{mg}$, respectively. Inhibition or induction of rat brain CYP2B can make doses of propofol active or inactive, respectively, indicating that altered CNS CYP2B activity can cause significant shifts in the apparent dose response for propofol.

\section{DISCUSSION}

This is the first study to show that localized metabolism of a centrally active drug in the brain can alter its pharmacological effect. We manipulated the activity of rat brain CYP2B by local enzyme inhibition or induction, which increased or decreased propofol-induced sleep times accordingly. We also showed that the concentrations of propofol in the brain correlated with the duration of sleep time. Our findings clearly show that CYP2B enzymatic activity in the rat brain significantly contributes to the metabolism of propofol and the resulting effect of the drug.

Some factors contributing to our inability to previously establish the role of CYP-mediated metabolism in the brain include: our inability to distinguish hepatic metabolism from that in the brain, lack of techniques to selectively alter brain metabolism alone, and the heterogeneity of the brain cellular and regional environments. This is further compounded by the great variability across these brain regions and cell types in CYP expression and inducibility (Miksys and Tyndale, 2004). To assess the contribution of brain CYPs to local drug metabolism and drug effect, we needed to establish a model where CYP2B activity could be altered across the entire brain without altering hepatic activity.
Using an i.c.v. injection of a CYP2B MBI, we were able to inhibit basal and induced rat brain CYP2B activity in two anatomically and functionally distinct brain regions, BS and FC, respectively, using a specific radiolabeling assay. These brain regions have significant decreases in brain glucose metabolism (a proxy measure for brain activity) during propofol anesthesia (Cavazzuti et al, 1991). The increase in brain, but not plasma, propofol concentrations as well as the strong correlation between brain levels and sleep response after an i.c.v. injection of a CYP2B MBI provide strong evidence that the altered propofol response is owing to the inhibition of brain, and not hepatic, CYP2B-mediated metabolism. In addition, the lack of effect of the i.c.v. injection of the CYP2B MBI on hepatic metabolism by CYP2B is further evidence for the selective inhibition of brain CYP2B. Genetically slow metabolizers for CYP2B, or individuals exposed to CYP2B inhibitors, might have higher levels of CYP2B in the brain, and would require lower levels to maintain anesthesia, as evidenced by the left-ward shift in the propofol response curve in the inhibited animals.

We have previously detected CYP2B in the human brain and found higher levels of CYP2B in the brains of human smokers (Miksys et al, 2003). Consistent with a role for induced rat brain CYP2B in reducing propofol response, there are also some case reports suggesting that smokers require higher doses of propofol to achieve loss of consciousness (Lysakowski et al, 2006), and that fewer smokers reported symptoms of postoperative nausea and vomiting (Chimbira and Sweeney, 2000). Alcoholics also have higher brain CYP2B levels compared with nonalcoholics (Miksys et al, 2003). Alcoholics required higher induction doses to achieve anesthesia compared with nonalcoholics, but plasma propofol levels did not differ significantly between the two groups at loss of consciousness (Fassoulaki et al, 1993). This provides indirect evidence to suggest that people (or animals) with higher brain CYP2B may require greater doses of propofol to achieve anesthesia, consistent with induced rat brain CYP2B shifting the propofol dose-response curve to the right. Similar to the lack of effect of chlorisondamine or mecamylamine in this study, varenicline, used in smoking cessation, and other nAChR blockers should not affect propofol response. The dose-dependent reversal of the reduction in sleep time by $\mathrm{C} 8-\mathrm{X}$ and the subsequent increases in brain propofol concentrations (which correlate with sleep time) also suggest that the effects seen here are owing to changes in local propofol concentrations, and are unlikely to be affected by altered receptor-mediated responses. Although the effects of induction and inhibition of brain CYP2B on propofol response were robust, and were unaffected by nAChR blockade, it is possible that in addition to binding to CYP2B, the two structurally diverse MBIs may have additional pharmacological targets, which were not examined in this study.

Our previous investigation of rat brain CYP2B showed a diurnal pattern of expression of rat brain CYP2B with higher levels during the night phase (Khokhar et al, 2010). Consistent with this finding, rats receiving an i.p. injection of propofol at night-time sleep for a shorter duration compared with animals tested with propofol during the daytime, which could possibly be due to faster propofol metabolism (Challet et al, 2007). Thus, variation in brain 
CYP2B levels may introduce noise or error into rat studies, if the drugs tested are CYP2B substrates and the experiments vary in the time of day. It is not known if there is a diurnal pattern of human brain CYP2B6 expression.

Our study employed an i.p. route of administration for propofol in contrast with the commonly used i.v. infusion route that is used in humans (Sebel and Lowdon, 1989). This route was chosen owing to its ease of use, and compared with an i.v. infusion, it is a less stressful and invasive procedure for the animals. One advantage of this method of administration is that the drug is susceptible to first-pass metabolism by the liver (Lukas et al, 1971); thus, if we show a role for CNS metabolism in pharmacological response using i.p. administration, it suggests this may also be the case for other drugs delivered orally. Even with a larger contribution of hepatic metabolism owing to the i.p. route of administration, alterations in brain CYP2B levels affected local propofol concentrations and response, indicating that these CYPs in the brain can act in addition to those in the liver, and alter drug response.

Although it is not clear if these findings have direct clinical relevance to human propofol anesthesia, they do suggest that CYPs in the rat brain are active and can alter drug effects through local brain metabolism. A variety of drug-metabolizing CYPs, including CYP2B, CYP2D, CYP2E1, CYP3A, and CYP4, have been detected in the brain (Meyer and Gehlhaus, 2010; Strobel et al, 2001) and their substrates include a wide range of centrally active drugs including opiate, antipsychotic, and antidepressant medications; thus, the impact of brain CYPs may be an important determinant of drug response. Local CYPmediated metabolism of these CNS acting drugs could have a direct impact on drug efficacy and could also contribute to drug tolerance, as seen from the shifts in propofol doseresponse curve following selective brain CYP2B manipulation. The ability of CYPs to metabolize endogenous neurosubstrates, such as serotonin (Fradette et al, 2004) and dopamine (Bromek et al, 2010), suggests that these CYPs have a role in normal brain function as well as in xenobiotic metabolism. Associations between genetic variation in CYP2D6 and resting brain activity (Kirchheiner et al, 2010) and personality scores (Bijl et al, 2009) support this possibility. CYPs inactivate and activate neurotoxins and procarcinogens, including chlorpyrifos and a variety of tobacco-specific nitrosamines (Ekins et al, 2008). Combined with the region-specific regulation of CYPs by commonly used drugs such as alcohol and nicotine, exposure to, and subsequent metabolism of, these toxins could contribute to local region-specific neurotoxicities or carcinomas. Phenobarbital, which can induce both hepatic and brain CYP2B, potentiated the neurotoxicity resulting from 9-methoxy$N$-2-methylellipticinium acetate (Upadhya et al, 2002). Recently, we have shown in a human neuronal cell line that toxicity following 1-methyl-4-phenylpyridinum was increased with the inhibition of CYP2D, an enzyme that can metabolically inactive this potent neurotoxin (Mann and Tyndale, 2010). Recent findings have also suggested a role for brain CYPs in altering the drug-hormone cross-talk in the brain, and found that neuroactive drugs that induce brain CYPs are associated with alterations in the side-effect profiles of these drugs and, in some cases exacerbate, the disease condition (Meyer and Gehlhaus, 2010).
Although previous studies have shown that CYP enzymes are active (Miksys and Tyndale, 2009) and inducible (Miksys and Tyndale, 2004) in the brain, there was no indication of whether their levels in the brain were sufficient to alter local drug metabolism and resulting drug effects. Here we provide strong evidence supporting a role for local drug metabolism by brain CYPs in altering the pharmacological actions of drugs. Centrally acting drugs can show large interindividual variability in response, which often does not correlate with plasma drug levels (Michels and Marzuk, 1993). Thus, localized CNS metabolism, which does not alter plasma levels as shown here, could contribute to variation in central drug response and potentially adverse drug reactions.

\section{ACKNOWLEDGEMENTS}

We would like to thank Drs Philip G Williams, Hiromi Morimoto, and William $\mathrm{F}$ Trager for generously providing us the radiolabeled 8-methoxypsoralen and Dr Howard Kaplan for his statistical guidance. We would also like to thank Fariba Baghai Wadji and Dr Bin Zhao for their technical assistance and Dr Sharon Miksys for her scientific consultation. This work was funded by the Centre for Addiction and Mental Health, CIHR MOP 97751, and a Canada Research Chair to Rachel F Tyndale.

\section{DISCLOSURE}

Dr Rachel F Tyndale is a shareholder in Nicogen Research, a company focused on the development of novel smoking cessation treatment approaches. None of the data contained in this manuscript alters or improves any commercial aspect of Nicogen and no Nicogen funds were used in this work. The manuscript was not reviewed by anyone else associated with Nicogen. Jibran Y Khokhar has no conflict of interest to declare.

\section{REFERENCES}

Albores A, Ortega-Mantilla G, Sierra-Santoyo A, Cebrian ME, Munoz-Sanchez JL, Calderon-Salinas JV et al (2001). Cytochrome P450 2B (CYP2B)-mediated activation of methylparathion in rat brain extracts. Toxicol Lett 124: 1-10.

Altomare C, Trapani G, Latrofa A, Serra M, Sanna E, Biggio G et al (2003). Highly water-soluble derivatives of the anesthetic agent propofol: in vitro and in vivo evaluation of cyclic amino acid esters. Eur J Pharm Sci 20: 17-26.

Bhargava VK, Saha L (2001). Cholinergic mechanism in imipramine and morphine antinoception. Boll Chim Farm 140: 201-204.

Bijl MJ, Luijendijk HJ, van den Berg JF, Visser LE, van Schaik RH, Hofman A et al (2009). Association between the CYP2D6*4 polymorphism and depression or anxiety in the elderly. Pharmacogenomics 10: 541-547.

Bromek E, Haduch A, Daniel WA (2010). The ability of cytochrome P450 2D isoforms to synthesize dopamine in the brain: an in vitro study. Eur J Pharmacol 626: 171-178.

Cavazzuti M, Porro CA, Barbieri A, Galetti A (1991). Brain and spinal cord metabolic activity during propofol anaesthesia. Br J Anaesth 66: 490-495.

Challet E, Gourmelen S, Pevet P, Oberling P, Pain L (2007). Reciprocal relationships between general (propofol) anesthesia 
and circadian time in rats. Neuropsychopharmacology 32: 728-735.

Chimbira W, Sweeney BP (2000). The effect of smoking on postoperative nausea and vomiting. Anaesthesia 55: 540-544.

Clarke PB, Chaudieu I, el-Bizri H, Boksa P, Quik M, Esplin BA et al (1994). The pharmacology of the nicotinic antagonist, chlorisondamine, investigated in rat brain and autonomic ganglion. Br J Pharmacol 111: 397-405.

Court MH, Duan SX, Hesse LM, Venkatakrishnan K, Greenblatt DJ (2001). Cytochrome P-450 2B6 is responsible for interindividual variability of propofol hydroxylation by human liver microsomes. Anesthesiology 94: 110-119.

Dam M, Ori C, Pizzolato G, Ricchieri GL, Pellegrini A, Giron GP et al (1990). The effects of propofol anesthesia on local cerebral glucose utilization in the rat. Anesthesiology 73: 499-505.

Ekins S, Iyer M, Krasowski MD, Kharasch ED (2008). Molecular characterization of CYP2B6 substrates. Curr Drug Metab 9: 363-373.

Fassoulaki A, Farinotti R, Mantz J, Desmonts JM (1994). Does tolerance develop to the anaesthetic effects of propofol in rats? Br J Anaesth 72: 127-128.

Fassoulaki A, Farinotti R, Servin F, Desmonts JM (1993). Chronic alcoholism increases the induction dose of propofol in humans. Anesth Analg 77: 553-556.

Favetta P, Degoute CS, Perdrix JP, Dufresne C, Boulieu R, Guitton J (2002). Propofol metabolites in man following propofol induction and maintenance. Br J Anaesth 88: 653-658.

Fradette C, Yamaguchi N, Du Souich P (2004). 5-Hydroxytryptamine is biotransformed by CYP2C9, 2C19 and 2B6 to hydroxylamine, which is converted into nitric oxide. $\mathrm{Br}$ J Pharmacol 141: 407-414.

Gervasini G, Carrillo JA, Benitez J (2004). Potential role of cerebral cytochrome $\mathrm{P} 450$ in clinical pharmacokinetics: modulation by endogenous compounds. Clin Pharmacokinet 43: 693-706.

Iohom $\mathrm{G}$, Ni Chonghaile $\mathrm{M}, \mathrm{O}^{\prime}$ Brien $\mathrm{JK}$, Cunningham $\mathrm{AJ}$, Fitzgerald DF, Shields DC (2007). An investigation of potential genetic determinants of propofol requirements and recovery from anaesthesia. Eur J Anaesthesiol 24: 912-919.

Kanto J, Gepts E (1989). Pharmacokinetic implications for the clinical use of propofol. Clin Pharmacokinet 17: 308-326.

Khokhar JY, Miksys SL, Tyndale RF (2010). Rat brain CYP2B induction by nicotine is persistent and does not involve nicotinic acetylcholine receptors. Brain Res 1348: 1-9.

Kirchheiner J, Seeringer A, Godoy AL, Ohmle B, Maier C, Beschoner P et al (2010). CYP2D6 in the brain: genotype effects on resting brain perfusion. Mol Psychiatry (e-pub ahead of print 6 April 2010; doi:10.138/mp.2010.42).

Koenigs LL, Trager WF (1998). Mechanism-based inactivation of cytochrome P450 2B1 by 8-methoxypsoralen and several other furanocoumarins. Biochemistry 37: 13184-13193.

Langley MS, Heel RC (1988). Propofol. A review of its pharmacodynamic and pharmacokinetic properties and use as an intravenous anaesthetic. Drugs 35: 334-372.

Le Guellec C, Lacarelle B, Villard PH, Point H, Catalin J, Durand A (1995). Glucuronidation of propofol in microsomal fractions from various tissues and species including humans: effect of different drugs. Anesth Analg 81: 855-861.

Lewis D (1996). Cytochromes P450. Structure, Function and Mechanism. Taylor \& Francis: Bristol. pp 122-123.

Liu SH, Wei W, Ding GN, Ke JD, Hong FX, Tian M (2009). Relationship between depth of anesthesia and effect-site concentration of propofol during induction with the targetcontrolled infusion technique in elderly patients. Chin Med J (Engl) 122: 935-940.

Ludbrook GL, Upton RN, Grant C, Gray EC (1996). Brain and blood concentrations of propofol after rapid intravenous injection in sheep, and their relationships to cerebral effects. Anaesth Intens Care 24: 445-452.

Lukas G, Brindle SD, Greengard P (1971). The route of absorption of intraperitoneally administered compounds. J Pharmacol Exp Ther 178: $562-564$.

Lysakowski C, Dumont L, Czarnetzki C, Bertrand D, Tassonyi E, Tramer MR (2006). The effect of cigarette smoking on the hypnotic efficacy of propofol. Anaesthesia 61: 826-831.

Mann A, Tyndale RF (2010). Cytochrome P450 2D6 enzyme neuroprotects against 1-methyl-4-phenylpyridinium toxicity in SH-SY5Y neuronal cells. Eur J Neurosci 31: 1185-1193.

Meyer RP, Gehlhaus M (2010). A role for CYP in the drughormone crosstalk of the brain. Expert Opin Drug Metab Toxicol 6: 675-687.

Michels R, Marzuk PM (1993). Progress in psychiatry (1). N Engl J Med 329: $552-560$.

Miksys S, Lerman C, Shields PG, Mash DC, Tyndale RF (2003). Smoking, alcoholism and genetic polymorphisms alter CYP2B6 levels in human brain. Neuropharmacology 45: 122-132.

Miksys S, Tyndale RF (2004). The unique regulation of brain cytochrome P450 2 (CYP2) family enzymes by drugs and genetics. Drug Metab Rev 36: 313-333.

Miksys S, Tyndale RF (2009). Brain drug-metabolizing cytochrome $\mathrm{P} 450$ enzymes are active in vivo, demonstrated by mechanism-based enzyme inhibition. Neuropsychopharmacology 34: 634-640.

Miksys SL, Tyndale RF (2002). Drug-metabolizing cytochrome P450s in the brain. J Psychiatry Neurosci 27: 406-415.

Nakayama H, Okuda H, Nakashima T, Imaoka S, Funae Y (1993). Nicotine metabolism by rat hepatic cytochrome P450s. Biochem Pharmacol 45: 2554-2556.

Paxinos G, Watson C (1986). The Rat Brain in Stereotaxic Coordinates, 2nd edn. Academic Press: San Diego.

Sebel PS, Lowdon JD (1989). Propofol: a new intravenous anesthetic. Anesthesiology 71: 260-277.

Seno H, He YL, Tashiro C, Ueyama H, Mashimo T (2002). Simple high-performance liquid chromatographic assay of propofol in human and rat plasma and various rat tissues. $J$ Anesth 16: 87-89.

Shyr MH, Tsai TH, Tan PP, Chen CF, Chan SH (1995). Concentration and regional distribution of propofol in brain and spinal cord during propofol anesthesia in the rat. Neurosci Lett 184: 212-215.

Siu EC, Tyndale RF (2008). Selegiline is a mechanism-based inactivator of CYP2A6 inhibiting nicotine metabolism in humans and mice. J Pharmacol Exp Ther 324: 992-999.

Strobel HW, Thompson CM, Antonovic L (2001). Cytochromes P450 in brain: function and significance. Curr Drug Metab 2: 199-214.

Tassonyi E, Charpantier E, Muller D, Dumont L, Bertrand D (2002). The role of nicotinic acetylcholine receptors in the mechanisms of anesthesia. Brain Res Bull 57: 133-150.

Upadhya SC, Chinta SJ, Pai HV, Boyd MR, Ravindranath V (2002). Toxicological consequences of differential regulation of cytochrome p450 isoforms in rat brain regions by phenobarbital. Arch Biochem Biophys 399: 56-65.

Vallejo YF, Buisson B, Bertrand D, Green WN (2005). Chronic nicotine exposure upregulates nicotinic receptors by a novel mechanism. J Neurosci 25: 5563-5572.

Yanev S, Kent UM, Pandova B, Hollenberg PF (1999). Selective mechanism-based inactivation of cytochromes P-450 2B1 and P4502 B6 by a series of xanthates. Drug Metab Dispos 27: 600-604.

Yanev SG, Kent UM, Roberts ES, Ballou DP, Hollenberg PF (2000). Mechanistic studies of cytochrome P450 2B1 inactivation by xanthates. Arch Biochem Biophys 378: 157-166. 